\title{
A Review of the Intrinsic Heavy Quark Content of the Nucleon
}

\author{
S. J. Brodsky, ${ }^{1}$ A. Kusina, ${ }^{2}$ F. Lyonnet, ${ }^{3}$ I. Schienbein, ${ }^{2}$ H. Spiesberger, ${ }^{4,5}$ and R. Vogt ${ }^{6,7}$ \\ ${ }^{1}$ SLAC National Accelerator Laboratory, Stanford University, Stanford, CA 94301, USA \\ ${ }^{2}$ Laboratoire de Physique Subatomique et de Cosmologie, Université Grenoble-Alpes, CNRS/IN2P3, \\ 53 avenue des Martyrs, 38026 Grenoble, France \\ ${ }^{3}$ Southern Methodist University, Dallas, TX 75275, USA \\ ${ }^{4}$ PRISMA Cluster of Excellence, Institut für Physik, Johannes Gutenberg-Universität Mainz, 55099 Mainz, Germany \\ ${ }^{5}$ Centre for Theoretical and Mathematical Physics and Department of Physics, University of Cape Town, \\ Rondebosch 7700, South Africa \\ ${ }^{6}$ Nuclear and Chemical Sciences Division, Lawrence Livermore National Laboratory, Livermore, CA 94551, USA \\ ${ }^{7}$ Physics Department, University of California at Davis, Davis, CA 95616, USA \\ Correspondence should be addressed to I. Schienbein; schien@lpsc.in2p3.fr
}

Received 17 April 2015; Accepted 15 June 2015

Academic Editor: Gianluca Cavoto

Copyright (C) 2015 S. J. Brodsky et al. This is an open access article distributed under the Creative Commons Attribution License, which permits unrestricted use, distribution, and reproduction in any medium, provided the original work is properly cited. The publication of this article was funded by SCOAP

We present a review of the state of the art of our understanding of the intrinsic charm and bottom content of the nucleon. We discuss theoretical calculations, constraints from global analyses, and collider observables sensitive to the intrinsic heavy quark distributions. A particular emphasis is put on the potential of a high energy and high luminosity fixed target experiment using the LHC beams (AFTER@LHC) to search for intrinsic charm.

\section{Introduction}

The existence of a nonperturbative intrinsic heavy quark component in the nucleon is a rigorous prediction of Quantum Chromodynamics (QCD). An unambiguous experimental confirmation is still missing and would represent a major discovery. The goal of this paper is to summarize our current understanding of this subject with a particular focus on the potential of a high energy and high luminosity fixed target experiment using the LHC beams (AFTER@LHC) [1-4] to search for intrinsic charm.

Production processes sensitive to the intrinsic heavy quark distributions of protons and nuclei are among the most interesting hadronic physics topics that can be investigated with AFTER@LHC. In contrast to the familiar extrinsic contributions which arise from gluon splitting in perturbative QCD, the intrinsic heavy quarks have multiple connections to the valence quarks of the proton and thus are sensitive to its nonperturbative structure. For example, if the gluongluon scattering box diagram, $g g \rightarrow Q \bar{Q} \rightarrow g g$ (the analog of QED light-by-light scattering), is inserted into the proton self-energy, the cut of this amplitude generates five-quark Fock states of the proton $|u u d Q \bar{Q}\rangle$; see Figure 1.

Intrinsic strange, charm, and bottom quarks are thus a fundamental property of the wavefunctions of hadronic bound states [5-8]. While the extrinsic contributions to the heavy quark parton distribution functions (PDFs) are most important at low $x$ and depend logarithmically on the heavy quark mass $M_{\mathrm{Q}}$, the intrinsic heavy quark contributions are dominant at high $x$ and depend on $1 / M_{\mathrm{Q}}^{2}$. Because the extrinsic heavy quarks are generated by gluon splitting, their PDFs are always softer than those of the parent gluon by a factor of $(1-x)$. In contrast, the high $x$ intrinsic heavy quark contributions are kinematically dominated by the regime where the $|u u d Q \bar{Q}\rangle$ state is minimally off shell, corresponding to equal rapidities of the constituent quarks. The resulting momentum and spin distributions of the intrinsic $Q$ and $\bar{Q}$ can be distinct, for example, $s(x) \neq \bar{s}(x)$, since the comoving $u u d Q \bar{Q}$ quarks are sensitive to the global quantum numbers of the protons. 


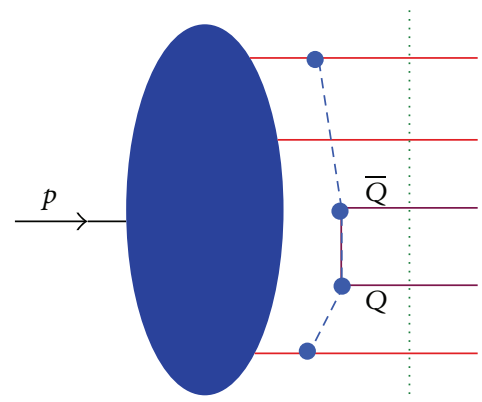

FIGURE 1: Five-quark Fock state $|u u d Q \bar{Q}\rangle$ of the proton and the origin of the intrinsic sea.

A finite intrinsic charm contribution to the nucleon has been extracted from lattice QCD. An analysis by the MILC collaboration [9] yields a probability for the charm matrix element $\langle N|c \bar{c}| N\rangle$ in the range of $5-6 \%$, consistent with a four-loop perturbative QCD calculation [10].

While the first experimental evidence of intrinsic heavy quarks came from the EMC measurement of the large $x$ charm structure function [11], a variety of other charm hadrons and charmonium measurements are consistent with the existence of intrinsic charm. Open charm observables in hadroproduction include forward $\Lambda_{c}$ production at the ISR [12]. Similarly, the coalescence of comoving $b, u$, and $d$ quarks from the $|u u d \bar{b} b\rangle$ intrinsic bottom Fock state in the proton can explain the high $x_{F}$ production of the $\Lambda_{b}(u d b)$ baryon, as observed at the ISR [12]. and asymmetries between leading and nonleading charms ( $\bar{D}$ mesons which share valence quarks with the projectile and $D$ mesons which do not, resp.) measured as functions of $x_{F}$ and $p_{T}$ in fixed target experiments, WA89 and WA82 at CERN; E791 and SELEX at Fermilab; see [13-15] and references therein. Previous fixed target $J / \psi$ measurements also give indications of important intrinsic charm contributions, particularly from the nuclear mass, or $A$, dependence, as measured by NA3 at CERN as well as E772 and, later, E866 at Fermilab; see, for example, [16]. Indeed, the $A$ dependence, proportional to $A^{\alpha}$, is quite different than the $\alpha \sim 1$ expected from extrinsic-type production [17]. At large $x_{F}$, there are indications of $A^{2 / 3}$ dependence, consistent with a nuclear surface-type interaction instead of the volume dependence of $\mathrm{PQCD}$. In addition, the NA3 collaboration measured double $J / \psi$ production at forward $x_{F}$ in $\pi A$ interactions, difficult to explain without an intrinsic charm mechanism [18]. All of these observables can be studied with higher energies and luminosities at AFTER@LHC, making precision measurements possible for the first time.

In addition to the typical observables for intrinsic heavy quarks, these intrinsic heavy quarks also contribute to a number of more exotic observables and inclusive and diffractive Higgs production $p p \rightarrow p p H$, in which the Higgs boson carries a significant fraction of the projectile proton momentum $[19,20]$. There are also important implications for intrinsic charm and bottom quarks in Standard Model physics, as in the weak decays of the $B$-meson [21] and a novel solution to the $J / \psi \rightarrow \rho \pi$ problem [22].AFTER@LHC could also shed light on these topics.

The rest of this paper is organized as follows. In Section 2, we give an overview of the theoretical models predicting the $x$-shape (but not the normalization) of the intrinsic charm and bottom parton distribution functions. In Section 3, we discuss the constraints on the normalization of the intrinsic charm (IC) obtained in global analyses of PDFs. Section 4 is devoted to the intrinsic bottom (IB) content of the nucleon, for which there are currently no quantitative constraints. In Section 5 we review collider observables sensitive to an intrinsic charm or bottom PDF. Finally, in Section 6 we present our conclusions.

\section{Theoretical Models}

The QCD wavefunction of a hadron can be represented as a superposition of quark and gluon Fock states. For example, at fixed light-front time, a hadron wavefunction can be expanded as a sum over the complete basis of free quark and gluon states: $\left|\Psi_{h}\right\rangle=\sum_{m}|m\rangle \psi_{m / h}\left(x_{i}, k_{T, i}\right)$ where the color-singlet states, $|m\rangle$, represent the fluctuations in the hadron wavefunction with the Fock components $\left|q_{1} q_{2} q_{3}\right\rangle$, $\left|q_{1} q_{2} q_{3} g\right\rangle,\left|q_{1} q_{2} q_{3} c \bar{c}\right\rangle$, and so forth. The boost-invariant light-front wavefunctions, $\psi_{m / h}\left(x_{i}, k_{T, i}\right)$, are functions of the relative momentum coordinates $x_{i}=k_{i}^{+} / P^{+}$and $k_{T, i}$ where $k_{i}$ denotes the parton momenta and $P$ the hadron momentum. Momentum conservation demands $\sum_{i=1}^{n} x_{i}=1$ and $\sum_{i=1}^{n} \vec{k}_{T, i}=0$ where $n$ is the number of partons in state $|m\rangle$. For example, as predicted by Brodsky and collaborators, in the BHPS model intrinsic charm fluctuations $[5,23]$ can be liberated by a soft interaction which breaks the coherence of the Fock state [24] provided the system is probed during the characteristic time that such fluctuations exist.

Microscopically, the intrinsic heavy quark Fock component in the proton wavefunction, $|u u d c \bar{c}\rangle$, is generated by virtual interactions such as $g g \rightarrow Q \bar{Q}$ where the gluons couple to two or more valence quarks. The probability for $c \bar{c}$ fluctuations to exist in a hadron is higher twist since it scales as $1 / m_{c}^{2}$ relative to the extrinsic, EC, leading-twist production by photon-gluon fusion [18].

The dominant Fock state configurations are not far off shell and thus have minimal invariant mass, $M^{2}=\sum_{i}^{n} \widehat{m}_{i}^{2} / x_{i}$, where $\widehat{m}_{i}^{2}=m_{i}^{2}+\left\langle\vec{k}_{T, i}^{2}\right\rangle$ is the square of the average transverse mass of parton $i$. The general form of the Fock state wavefunction for a hadron with mass $m_{h}$ appropriate to any frame at fixed light-front time is

$$
\Psi\left(x_{i}, \vec{k}_{\perp i}\right)=\frac{\Gamma\left(x_{i}, \vec{k}_{\perp i}\right)}{m_{h}^{2}-M^{2}},
$$

where $\Gamma$ is a vertex function, expected to be a slowly varying, decreasing function of $m_{h}^{2}-M^{2}$. The particle distributions are then controlled by the light-front energy denominator and phase space. This form for the higher Fock components is applicable to an arbitrary number of light and heavy partons. Intrinsic $c \bar{c}$ Fock components with minimum invariant mass correspond to configurations with equal rapidity 
constituents. Thus, unlike extrinsic heavy quarks generated from a single parton, intrinsic heavy quarks carry a larger fraction of the parent momentum than the light quarks in the state $[5,23]$.

The parton distributions reflect the underlying shape of the Fock state wavefunction. Assuming that it is sufficient to use $\left\langle k_{T}^{2}\right\rangle$ for the transverse momentum, the probability distribution as a function of $x$ in a general $n$-particle intrinsic $c \bar{c}$ Fock state is

$$
\frac{d P_{\mathrm{IC}}}{d x_{i} \cdots d x_{n}}=N_{n} \frac{\delta\left(1-\sum_{i=1}^{n} x_{i}\right)}{\left(m_{h}^{2}-\sum_{i=1}^{n}\left(\widehat{m}_{i}^{2} / x_{i}\right)\right)^{2}},
$$

where $N_{n}$ normalizes the $n$-particle Fock state probability.

At LO in the heavy quark limit, $\widehat{m}_{c}, \widehat{m}_{\bar{c}} \gg m_{h}, \widehat{m}_{q}$,

$$
\frac{d P_{\mathrm{IC}}}{d x_{i} \cdots d x_{n}}=N_{n} \frac{x_{c}^{2} x_{\bar{c}}^{2}}{\left(x_{c}+x_{\bar{c}}\right)^{2}} \delta\left(1-\sum_{i=1}^{n} x_{i}\right)
$$

leading to

$$
\begin{aligned}
F_{2 c}^{\mathrm{ICLO}}(x) & =\frac{8}{9} x c(x) \\
& =\frac{8}{9} x \int d x_{1} \cdots d x_{\bar{c}} \frac{d P_{\mathrm{IC}}}{d x_{i} \cdots d x_{\bar{c}} d x_{c}} .
\end{aligned}
$$

There are many applications of intrinsic charm in charm hadron production. See, for example, [13-16, 18] for more details.

Paiva et al. have also calculated an intrinsic charm component of the nucleon sea within the context of the meson cloud model [25]. They assumed that the nucleon can fluctuate into $\bar{D} \Lambda_{c}$. The $\bar{c}$ distribution in the nucleon is then

$$
x \bar{c}_{N}(x)=\int_{x}^{1} d y f_{\bar{D}}(y) \frac{x}{y} \bar{c}_{\bar{D}}\left(\frac{x}{y}\right),
$$

where

$$
f_{\bar{D}}(y)=\frac{g_{\bar{D} N \Lambda_{c}}^{2}}{16 \pi^{2}} y \int_{-\infty}^{t_{\max }} d t \frac{\left[-t+\left(m_{\Lambda_{c}}-m_{N}\right)^{2}\right]}{\left[t-m_{\bar{D}}^{2}\right]^{2}} F^{2}(t)
$$

with $F(t)$ being a form factor at the $D N \Lambda$ vertex and $t_{\max }=$ $m_{N}^{2} y-m_{\Lambda_{c}}^{2} y /(1-y)$. In this case they chose a monopole form factor with $\Lambda_{m}=1.2 \mathrm{GeV}$. The coupling constant was assumed to be $g_{\bar{D} N \Lambda_{c}}=-3.795$. From heavy quark effective theories [26], the $\bar{c}$ distribution in the $\bar{D}$ is expected to be hard because, in the bound state, the $\bar{c}$ exchanges momenta much less than $m_{c}$. They make the extreme assumption that the entire $\bar{D}$ momentum is carried by the charm quark, $\bar{c}_{\bar{D}}=$ $x \delta(x-y)$.

Next, Steffens et al. investigated all the charm structure function data with two variants of intrinsic charm [27]. The first was that of (4), called IC1 in their paper, while the second was a meson cloud model, IC2. In the second approach, the $\bar{c}$ distribution is obtained from the light-front distribution of $\bar{D}^{0}$ mesons in the nucleon:

$$
\begin{gathered}
\bar{c}^{\mathrm{IC} 2}(x) \approx f_{\bar{D}}(x)=\frac{1}{16 \pi^{2}} \int_{0}^{\infty} d k_{\perp}^{2} \\
\cdot \frac{g^{2}\left(x, k_{\perp}^{2}\right)}{x(1-x)\left(s_{\bar{D} \Lambda_{c}}-m_{N}^{2}\right)^{2}} \\
\cdot \frac{k_{\perp}^{2}+\left(m_{\Lambda_{c}}-(1-x) m_{N}\right)^{2}}{1-x} .
\end{gathered}
$$

A hard charm momentum distribution was assumed in the $\bar{D}$, similar to that of [25]. The vertex function $g^{2}\left(x, k_{\perp}^{2}\right)$ is parameterized as $g^{2}=g_{0}^{2}\left(\Lambda^{2}+m_{N}^{2}\right) /\left(\Lambda^{2}+s_{\bar{D} \Lambda_{c}}\right)$ where $s_{\bar{D} \Lambda_{c}}$ is the square of the center-of-mass energy of the $\bar{D} \Lambda_{c}$ system and $g_{0}^{2}$ the coupling constant at $s_{\bar{D} \Lambda_{c}}=m_{N}^{2}$. For an intrinsic charm probability of $1 \%, \Lambda \approx 2.2 \mathrm{GeV}$. The charm distribution is then

$$
c^{\mathrm{IC} 2}(x) \approx \frac{3}{2} f_{\Lambda_{c}}\left(\frac{3 x}{2}\right),
$$

where the charm distribution in the $\Lambda_{c}$ is assumed to be $c_{\Lambda_{c}} \sim$ $\delta(x-2 / 3)$ and $f_{\Lambda_{c}}(x)=f_{\bar{D}}(1-x)$.

Pumplin [28] considered a model where a point scalar particle of mass $m_{0}$ couples with strength $g$ to $N$ scalar particles with mass $m_{1}, m_{2}, \ldots, m_{N}$. The probability density is then

$$
\begin{aligned}
d P= & \frac{g^{2}}{\left(16 \pi^{2}\right)^{N-1}(N-2) !} \prod_{j=1}^{N} d x_{j} \delta\left(1-\sum_{j=1}^{N} x_{j}\right) \int_{s_{0}}^{\infty} d s \\
& \cdot \frac{\left(s-s_{0}\right)^{N-2}}{\left(s-m_{0}^{2}\right)^{2}}|F(s)|^{2},
\end{aligned}
$$

where $s_{0}=\sum_{j=1}^{N}\left(m_{j}^{2} / x_{j}\right)$. The form factor $F(s)$ suppresses higher mass state contributions. If the quark transverse momenta are neglected, with $m_{c}$ much greater than all other mass scales, and $F(s)=1$, then the BHPS model is recovered. Two types of form factors were studied, an exponential $|F(s)|^{2}=\exp \left[-\left(s-m_{0}^{2}\right) / \Lambda^{2}\right]$ and a power law $|F(s)|^{2}=$ $1 /\left(s+\Lambda^{2}\right)^{n}$ where the cutoff $\Lambda$ is varied between 2 and $10 \mathrm{GeV}$.

Hobbs et al. employed a meson cloud type approach but specified the spin and parity of all lowest mass charm meson-baryon combinations from the 5-particle $|u u d c \bar{c}\rangle$ Fock states of the proton [29]. They pointed out that treating quarks as scalar point-like particles, as in, for example, [28], does not conserve spin and parity. They calculated the appropriate meson-baryon splitting functions for the mesonbaryon combinations and found that the production of charm mesons would be almost entirely through $D^{*}$ mesons. To study the phenomenological distributions of charm mesons and baryons in this approach, they studied exponential and confining vertex functions, $\propto \exp \left[-\left(s-m_{D}^{2}\right) / \Lambda^{2}\right]$ and $(s-$ $\left.m_{D}^{2}\right) \exp \left[-\left(s-m_{D}^{2}\right) / \Lambda^{2}\right]$, respectively. They used these results to compare to the $\Lambda_{c}$ distribution from the ISR [30] and the $\Lambda_{c} / \bar{\Lambda}_{c}$ asymmetry from SELEX [31]. See [29] for details. 


\section{Global Analyses of PDFs with Intrinsic Charm}

In the standard approach employed by almost all global analyses of PDFs, the heavy quark distributions are generated radiatively, according to DGLAP evolution equations [3234], starting with a perturbatively calculable boundary condition $[35,36]$ at a scale of the order of the heavy quark mass. In other words, there are no free fit parameters associated with the heavy quark distribution and it is entirely related to the gluon distribution function at the scale of the boundary condition. As a consequence, also the PDF uncertainties for the heavy quark and the gluon PDFs are strongly correlated as has been discussed in the context of inclusive Higgs production at the Tevatron and the LHC [37]. However, a purely perturbative treatment might not be adequate in particular for the charm quark with a mass $m_{c} \simeq 1.3 \mathrm{GeV}$ which is not much bigger than typical hadronic scales but also for the bottom quark with a mass $m_{b} \simeq 4.5 \mathrm{GeV}$. Indeed, as discussed above, light-front models predict a nonperturbative ("intrinsic") heavy quark component in the proton wavefunction $[5,23]$. Motivated by the theoretical predictions of the BHPS light-front model, analyses of the charm distribution in the proton going beyond the common assumption of purely radiatively generated charm date back almost as far as the BHPS predictions themselves. For definiteness, in the following we refer to the radiatively generated charm by $c_{0}(x, Q)$ and to the intrinsic charm by $c_{1}(x, Q)$. The full charm parton distribution is then given by the sum $c(x, Q)=c_{0}(x, Q)+c_{1}(x, Q)$. Strictly speaking, this decomposition is defined at the initial scale $Q_{0} \simeq m_{c}$ of the DGLAP evolution but holds to a good approximation at any scale since the intrinsic component $c_{1}$ is governed (to a very good approximation) by a standalone nonsinglet evolution equation [38]. A similar decomposition is understood for the bottom quark which will be discussed in Section 4 .

The BHPS model of the $|u u d c \bar{c}\rangle$ Fock state predicts a simple form for $F_{2 c}(x)$ :

$$
\begin{aligned}
& F_{2 c}^{\mathrm{IC}}(x)=\left(\frac{8}{9} x\right) \frac{1}{2} N_{5} x^{2}\left[\frac{1}{3}(1-x)\left(1+10 x+x^{2}\right)\right. \\
& +2 x(1+x) \ln x] .
\end{aligned}
$$

If there is a $1 \%$ intrinsic charm contribution to the proton PDF, $N_{5}=36$.

Hoffmann and Moore incorporated mass effects and introduced next-to-leading order corrections as well as scale evolution [39]. They compared their result to the EMC $F_{2 c}$ data from muon scattering on iron at high $x$ and $Q^{2}$ with the intrinsic charm contribution added to the leading order calculation of $F_{2 c}$ by photon-gluon fusion.

A complete next-to-leading order analysis of both the "extrinsic" radiatively generated charm component and the intrinsic component was later carried out by Harris et al. [7]. The EMC data with $\bar{\nu}=\overline{Q^{2}} / 2 m_{p} \bar{x}=53,95$, and $168 \mathrm{GeV}$ were fit by a sum of the extrinsic and intrinsic components
[7]. The normalization of the two components was left as free parameters:

$$
F_{2 c}\left(x, \mu^{2}, m_{c}^{2}\right)=\epsilon F_{2 c}^{\gamma p}\left(x, \mu^{2}, m_{c}^{2}\right)+\delta F_{2 c}^{\mathrm{IC}}\left(x, \mu^{2}, m_{c}^{2}\right),
$$

with the scale $\mu=\sqrt{m_{c \bar{c}}^{2}+Q^{2}}$. The parameter $\epsilon$, typically larger than unity, was considered to be an estimate of the NNLO contribution to the extrinsic contribution. Since a $1 \%$ normalization of the IC component was assumed in (11), the fitted value of $\delta$ is the fraction of this normalization. Given the quality of the data, no statement could be made about the intrinsic charm content of the proton when $\bar{v}=$ 53 and $95 \mathrm{GeV}$. However, with $\bar{v}=168 \mathrm{GeV}$ an intrinsic charm contribution of $(0.86 \pm 0.60) \%$ was indicated. These results were consistent with those of the original analysis by Hoffmann and Moore [39].

The BHPS light-front model assumes that $c_{1}(x)=\bar{c}_{1}(x)$. Meson cloud models, introduced later, treat the 5-particle Fock state as a combination of (predominantly) $\bar{D}^{0} \Lambda_{c}^{+}$. In this case, of course, $c_{1}(x) \neq \bar{c}_{1}(x)$ with the $\bar{c}$ quark in the $\bar{D}^{0}$ carrying more momentum than the $c$ quark in the charm baryon. An analysis by Steffens et al. in the context of the meson cloud model and using a hybrid scheme to interpolate between massless evolution at high $Q^{2}$ and "extrinsic" production at low $Q^{2}$ found a limit of $\sim 0.4 \%$ [27].

Regardless of whether or not the models predict $\bar{c}_{1}(x)-$ $c_{1}(x)>0$, intrinsic charm should provide the dominant contribution to the charm density in the proton at large $x$ [28].

For some time, no other analyses of the charm structure function were made. The EMC data remain the only measurement of the charm structure function in the relevant $\left(x, Q^{2}\right)$ regime and are the only DIS data cited as evidence for intrinsic charm. The HERA data on $F_{2 c}$ were at too low $x$ to address the issue.

The first global analyses of the proton PDFs with an intrinsic charm contribution included were performed by members of the CTEQ collaboration $[40,41]$. In addition to the BHPS and meson cloud approaches, they also allowed for a "sea-like" contribution with the same shape as the radiatively generated charm distribution. They characterized the magnitude of the intrinsic charm component $\left(c_{1}\left(x, Q^{2}\right)\right)$ by the first moment of the charm distribution at the input scale $Q_{0}=m_{c}=1.3 \mathrm{GeV}$ (Note that at $Q_{0}=m_{c}$ the radiatively generated charm component $\left(c_{0}\left(x, Q^{2}\right)\right)$ vanishes at NLO in the $\overline{\mathrm{MS}}$ scheme so that $c\left(x, Q_{0}^{2}\right)=c_{1}\left(x, Q_{0}^{2}\right)$.):

$$
c_{1}\left(N=1, Q_{0}^{2}\right)=\int_{0}^{1} d x c_{1}\left(x, Q_{0}^{2}\right)=0.01
$$

which translates into a momentum fraction

$$
\langle x\rangle_{c_{1}+\bar{c}_{1}}=\int_{0}^{1} d x x\left[c_{1}\left(x, Q_{0}^{2}\right)+\bar{c}_{1}\left(x, Q_{0}^{2}\right)\right]=0.0057 .
$$

They found that the global analyses of hard-scattering data provided no evidence for or against the existence of intrinsic charm up to $\langle x\rangle_{\mathcal{c}_{1}+\bar{c}_{1}}=0.0057$; that is, the quality of the fit is insensitive to $\langle x\rangle_{c_{1}+\bar{c}_{1}}$ in this interval. They also 
found that the allowed range was greatest for the sea-like IC expected since this shape is rather easily interchangeable with other sea quark components while the other, harder, charm distributions are not [40]. In addition, they concluded that the enhancement due to IC relative to analyses without it persisted up to scales of $\sim 100 \mathrm{GeV}$ and could have an influence on charm-initiated processes at the LHC, as is discussed later. The CTEQ6.6C proton PDFs were generated as a result of this analysis [41].

There are two recent updates to the global analyses, reaching different conclusions about the importance of intrinsic charm. The first, by Dulat et al. [42], follows the previous work in the context of the CTEQ collaboration [40,41]. The second, by Jimenez-Delgado et al. [43], included more lower energy data than the previous global analyses.

The result of Dulat et al. [42] was based on the CT10 NNLO parton densities. Here the strong coupling, $\alpha_{S}\left(Q^{2}\right)$, the evolution equations, and the matrix elements are calculated at NNLO. Only the inclusive jet data still required NLO expressions. Their analysis included DIS data from BCDMS, NMC, CDHSW, and CCFR; SIDIS data from NuTeV and CCFR; the combined DIS and $F_{2 c}$ data from HERA; Drell-Yan production; the $W$ charge asymmetry and $Z^{0}$ rapidity from $\mathrm{CDF}$ and $\mathrm{D} 0$; and the inclusive jet measurements from $\mathrm{CDF}$ and D0; see [42] for a complete list.

Two models of IC were considered: the BHPS light-front model and the sea-like IC introduced in [40]. They found a broader possible probability range for IC in this analysis, $\langle x\rangle_{\text {IC }}=\langle x\rangle_{c_{1}+\bar{c}_{1}}\left(Q_{0}^{2}\right) \lesssim 0.025$ for BHPS and $\langle x\rangle_{\text {IC }} \lesssim 0.015$ for the sea-like IC, summarized in Figure 2. This finding differs from the previous work which found a larger upper limit on IC for the sea-like model. They believe that the difference is caused by the improved treatment of the charm quark mass in the later study [42].

In addition to the global fit, they also tested the sensitivity of their result to individual experiments by introducing a penalty factor, $T_{2}(i)$, for each experiment $i$. This penalty factor is designed to increase more rapidly than the $\chi_{i}^{2}$ for that experiment when $\chi_{i}^{2}$ goes beyond the $90 \%$ confidence level. The penalty factor employs an equivalent Gaussian variable $S_{n}$ which measures the goodness of fit for each individual data set. Values of $S_{n} \leq|1|$ are considered good fits, $S_{n}>3$ is considered to be a poor fit, and values of $S_{n}<-3$ are better fits than expected from usual statistical analyses. Using the $S_{n}$ dependence on $\langle x\rangle_{\text {IC }}$, they determined which of the data sets used in the global analyses are most sensitive to intrinsic charm. The upper limit on the BHPS value of $\langle x\rangle_{\text {IC }}$ comes from the CCFR structure function data while the HERA combined charm data sets the upper limit on IC from the sealike model [42].

They also studied the sensitivity of their sea-like result to the charm quark mass and found that if the charm quark mass was raised from $1.3 \mathrm{GeV}$, as in the CT10 fits, to $1.67 \mathrm{GeV}$, then the minimum $\chi^{2}$ for the global analyses would support $\langle x\rangle_{\text {IC }}=0.01$ rather than 0 although the global $\chi^{2}$ is worse for the larger charm mass [42]. Finally, they showed how $W$ and $Z$ production at the LHC might be affected by a nonzero IC contribution.

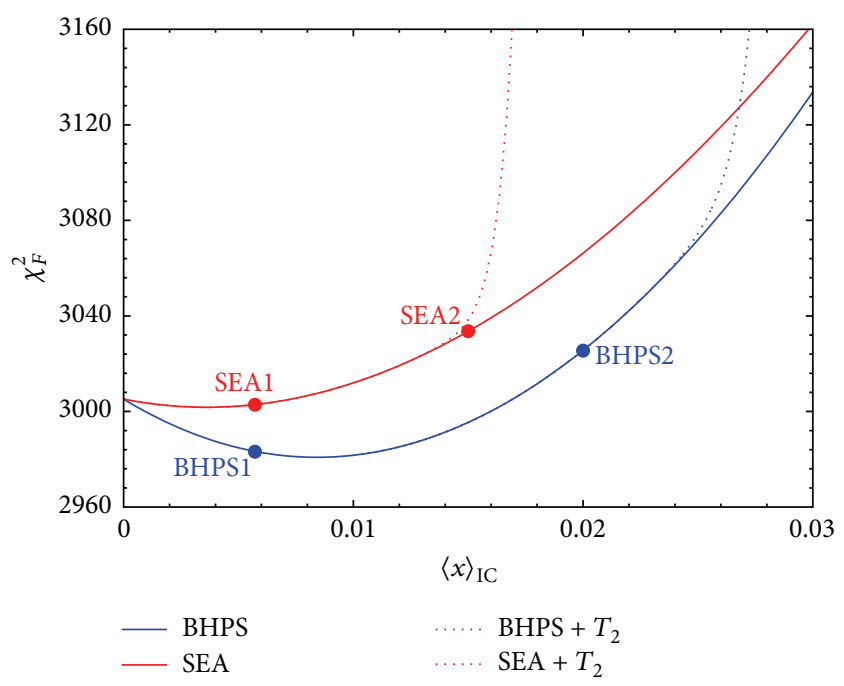

FIGURE 2: The global chi-square function versus charm momentum fraction $\langle x\rangle_{\text {IC }}$. The two curves are determined from fits with many values of $\langle x\rangle_{\text {IC }}$. Two exemplary fits for each IC model are shown as dots. Blue dots denote the BHPS model; the dots have $\langle x\rangle_{\text {IC }}=0.57 \%$ and $2 \%$, which are denoted as BHPS1 and BHPS2. Red denotes SEA model; the dots have $\langle x\rangle_{\text {IC }}=0.57 \%$ and $1.5 \%$, which are denoted as SEA1 and SEA2. Additionally the dotted lines show global chisquare function with additional penalty, $T_{2}(i)$, used to set the upper limits on the allowed IC component (figure taken from [42]).

In the most recent study, Jimenez-Delgado et al. [43] included the full range of high energy scattering data by using looser kinematic cuts $Q^{2} \geq 1 \mathrm{GeV}^{2}$ and $W^{2} \geq 3.5 \mathrm{GeV}^{2}$. In particular, they included the lower energy SLAC fixed target data which did not pass the more stringent standard DIS cuts on the $\left(Q^{2}, W^{2}\right)$ plane applied in the previous work [40-42]. The EMC $F_{2 c}$ data, cited as the strongest evidence for intrinsic charm in DIS, are used as a consistency check. The low energy, high $x$, fixed target data lie precisely in the region where IC is expected to be most important. Thus including these data could enhance the sensitivity of the global fit to IC. Note, however, that some of these newly added data are on heavier targets than the deuteron and thus target mass corrections, nuclear corrections for $A>2$, and higher-twist effects need to be taken into account [43].

They followed the framework of the JR14 [44] global fit which decomposed $F_{2}$ into light and heavy components. The charm component is itself separated into the "extrinsic" and intrinsic charm components. The fixed-flavor number scheme is used to compute the extrinsic contribution. In this scheme, the charm quark mass enters the PDF evolution only indirectly through the running of $\alpha_{s}$ [43]. They employed a charm quark mass of $1.3 \mathrm{GeV}$, as did Dulat et al. [42]. They used all three intrinsic charm models previously considered: BHPS, the meson cloud model (this time including pseudoscalar and vector mesons as well as spin $1 / 2$ and spin $3 / 2$ charm baryons-the CTEQ analyses only included the scalar $\bar{D} \Lambda_{c}$ fluctuation), and the sea-like component [43]. The IC contribution was evolved up to NLO. 


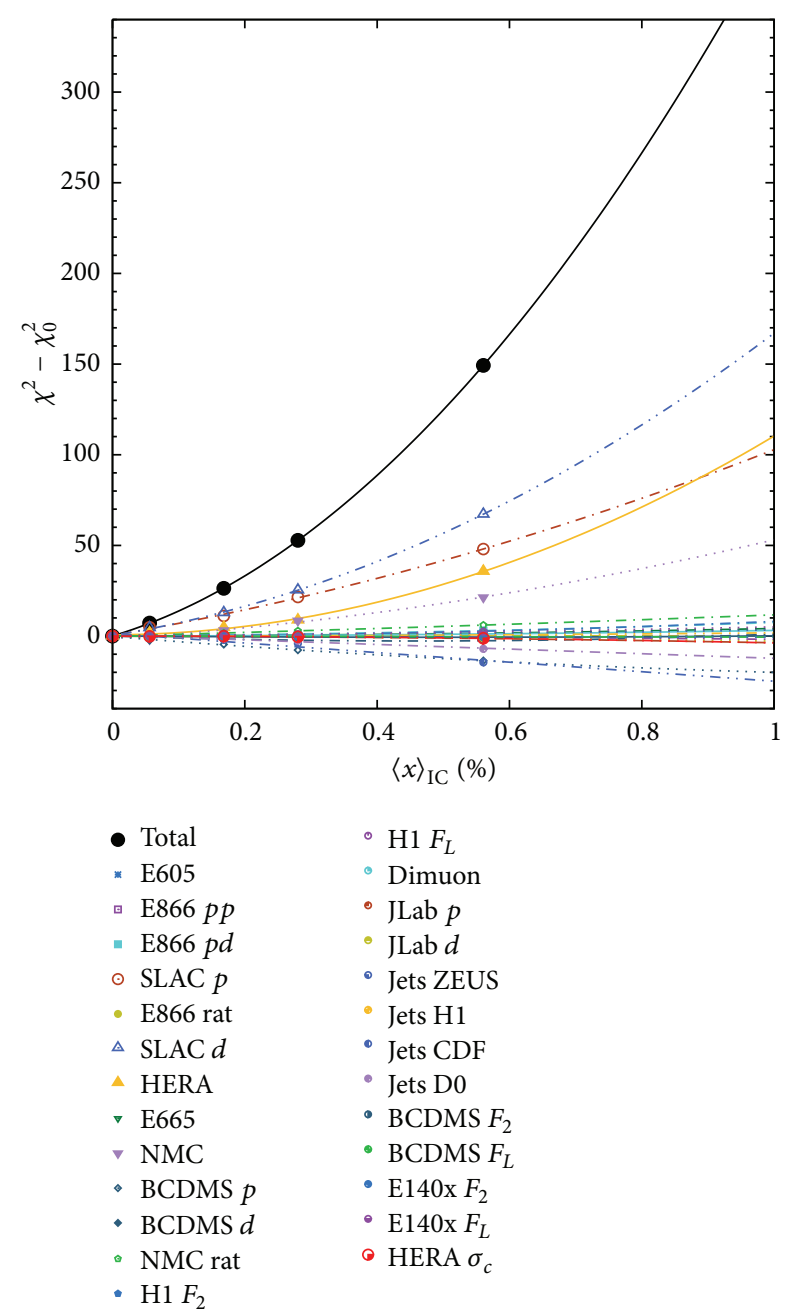

FIgUre 3: Contributions to the total $\chi^{2}$ (black circles), relative to the value $\chi_{0}^{2}$ for no IC, of various data sets as a function of the momentum fraction $\langle x\rangle_{\text {IC }}$ (figure taken from [43]).

They found that the total $\chi^{2}$ is minimized for $\langle x\rangle_{\text {IC }}=0$ with $\langle x\rangle_{\text {IC }}<0.1 \%$ at the $5 \sigma$ level. When a hadron suppression factor to suppress charm contributions near threshold is applied, they find a minimum $\chi^{2}$ at $\langle x\rangle_{\text {IC }}=(0.15 \pm 0.09) \%$ for the full data set. The SLAC $F_{2}$ (large $x$ ), NMC cross sections (medium $x$ ), and HERA $F_{2 c}$ (low $x$ ) display the greatest sensitivity to IC; see Figure 3 for details. However, fits without the SLAC data still give a low IC contribution [43]. The difference between their results and previous results is in part due to the very different tolerance criteria, $\Delta \chi^{2}=1$ for their fit and $\Delta \chi^{2}=100$ for Dulat et al. [42]. Increasing the tolerance to $\Delta \chi^{2}=100$ would also accommodate $\langle x\rangle_{\text {IC }}=1 \%$ at the $1 \sigma$ level [43]. (For a critical discussion of the analysis in [44] and in particular of the tolerance criterion $\Delta \chi^{2}=1$ see $[45,46]$.)

When checked against the EMC $F_{2 c}$ data, a clear preference for IC is found, as expected, for the highest $x$ data. (The EMC $\mu$ - $p$ data have not been included in some global analyses because of a possible conflict with HERA $e-p$ data at very low $x$; however, the relative suppression of the low $x$ and low $Q^{2}$ EMC data could be accounted for by nuclear shadowing, suppressed evolution, higher twist, and other effects.)

Given that the two most recent analyses set significantly different limits on IC, it is important to collect further large $x$ data, particularly on $F_{2 c}$ to try and place greater confidence on the limit of IC in the nucleon. This would be an important measurement at the future electron-ion collider.

\section{Predictions for Intrinsic Bottom}

In contrast to the case of intrinsic charm, there is currently no global analysis available that investigates the possibility of an intrinsic bottom (IB) content of the nucleon. The main reason for this is the lack of experimental data that could constrain it. The BHPS light-front model [5] predicts the existence of IB with an $x$-shape very similar to the one of IC given in (10) but with a normalization which is parametrically suppressed by the ratio $m_{c}^{2} / m_{b}^{2}$. This fact, together with the observation that the IB PDF is governed (to an excellent approximation) by an independent nonsinglet evolution equation [38], can be used to investigate IB in a flexible way without the need of a dedicated global analysis. Such a study has been done in [38] where a set of decoupled IB (and IC) PDFs has been provided and used together with the CTEQ6.6 PDFs [41] to estimate the impact of the IB on new physics searches at the LHC. The advantage of this approach is that the provided IB (IC) PDF can be used with any standard set of PDFs and the normalization of the intrinsic component can be freely adjusted. This is especially useful for studies of possible IB effects, as, in that case, there are no experimental limits on what amount of IB is allowed.

In the following we show some of the results found in [38]. In this work, the boundary condition for the IB distribution was modeled using the IC distributions in the CTEQ analyses [40, 41] scaled down by the mass factor $m_{c}^{2} / m_{b}^{2}$. The result of such an intrinsic bottom distribution $b_{1}\left(x, Q^{2}\right)$, with normalization $\int_{0}^{1} d x b_{1}\left(x, m_{c}^{2}\right)=0.01 \times m_{c}^{2} / m_{b}^{2}$, is shown in Figure 4, where the ratio of the intrinsic $\left(b_{1}\right)$ and the radiatively generated $\left(b_{0}\right)$ component of the bottom PDF is plotted. As always in the light-front models the intrinsic component is mostly present at large $x$ values. We can see that for low scales $Q \sim 10 \mathrm{GeV}$ the modification of the bottom PDF, $\kappa_{b}=1+b_{1} / b_{0}$, can reach $\kappa_{b}=2.5$. However, it decreases rapidly with the rising scale. Since $b_{1}$ evolves independently of the other PDFs the change in the normalization of the IB component in Figure 4 can be done by simply rescaling the curves in the figure. If we allowed for a $0.035 \times \mathrm{m}_{c}^{2} / \mathrm{m}_{b}^{2}$ normalization of the IB the modification of the bottom PDF would be given by $\kappa_{b}=1+b_{1} / b_{0} \times 3.5$, which for high $x$ and $Q \sim 10 \mathrm{GeV}$ would result in an enhancement of the bottom $\mathrm{PDF}$ by a factor $\sim 6.25$. However, at a scale of around $100 \mathrm{GeV}$ and $x$ below $0.2-0.3$, even with the higher IB normalization, the effect is becoming negligible.

In Figure 5 we show the sum of the intrinsic bottom PDF $b_{1}$ and the dynamically generated PDF $b_{0}$ from CTEQ6.6 for different normalizations of the IB component, namely, 0.01 and $0.035 \times m_{c}^{2} / m_{b}^{2}$. We compare this sum to the asymmetric 


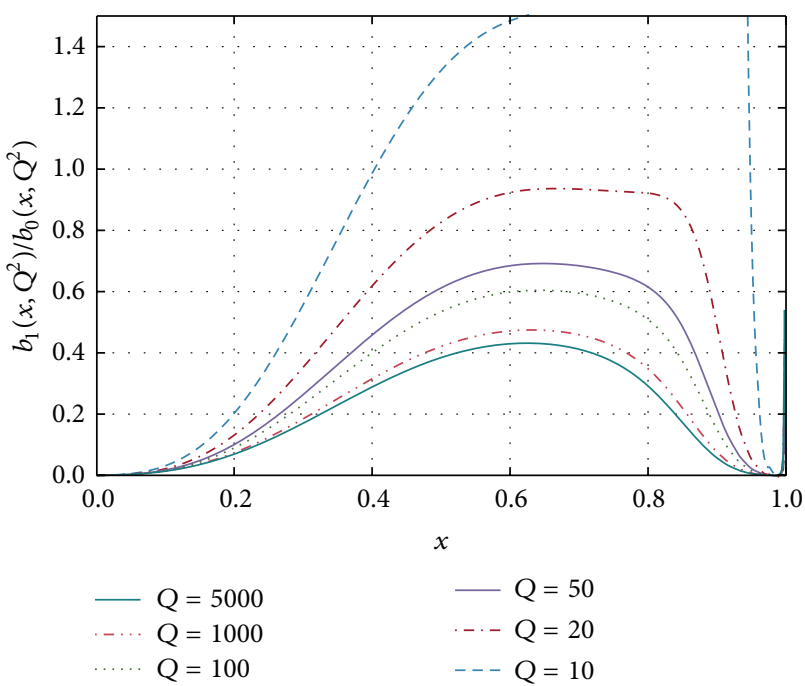

FIGURE 4: Ratio of intrinsic $\left(b_{1}\right)$ and dynamically generated $\left(b_{0}\right)$ bottom PDFs for various $Q$ scales. The perturbative bottom PDF from CTEQ6.6c0 [41] is used; the normalization of the IB is taken to be such that $\int_{0}^{1} d x b_{1}\left(x, m_{c}^{2}\right)=0.01 \times m_{c}^{2} / m_{b}^{2}$ (figure taken from [38]).

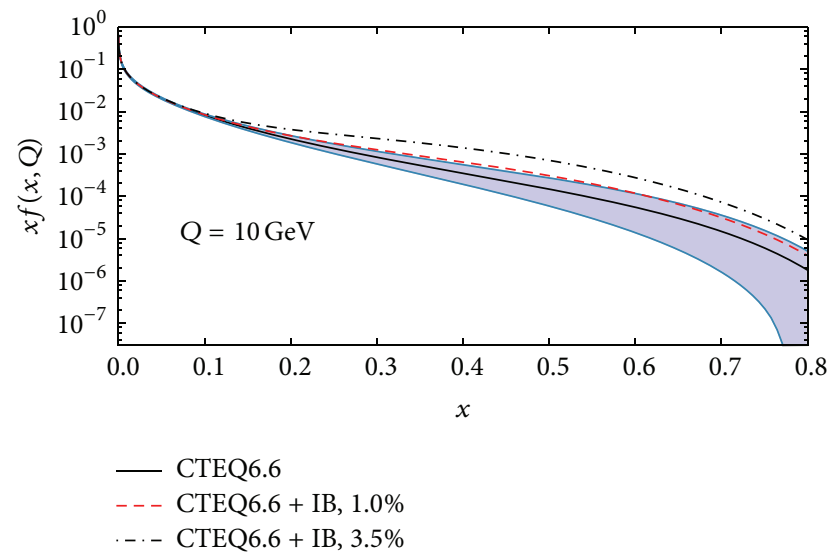

(a)

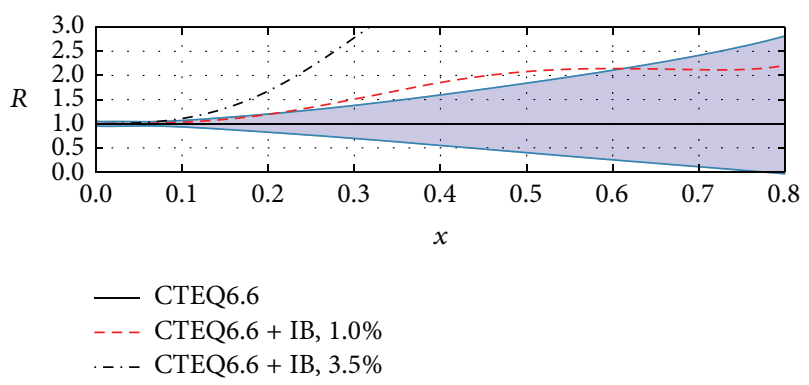

(b)

FIgURE 5: CTEQ6.6 $+b_{1}$ for different normalizations of the intrinsic bottom quark PDF at the scale $Q=10 \mathrm{GeV}$, compared to the asymmetric PDF errors from the same set (a). Also shown is the ratio of the same PDF sets to the central value of CTEQ6.6 (b). uncertainties (The asymmetric errors are computed following $[47,48]$.) of the CTEQ6.6 PDF set (a). In the same figure is also shown the ratio of the same PDFs to the central value of CTEQ6.6 (b). As can be seen, the IB curve with the $0.035 \times$ $m_{c}^{2} / m_{b}^{2}$ normalization clearly lies outside the uncertainty band whereas the one with the smaller normalization is marginally outside the band (up to $x \leqslant 0.6$ ).

If we are looking for new physics with couplings proportional to the mass, the suppression of IB compared to the IC would be partly compensated by the square of the coupling. For a more detailed study of the relevant partonparton luminosities please see [38].

\section{Collider Observables}

Several collider observables receive large contributions from heavy quark initiated subprocesses and are hence potentially sensitive to an intrinsic charm content in the nucleon. In order to expect optimal effects the heavy quark PDF should be probed at large $x \gtrsim 0.2$ (for light-front models) and not too large factorization scales. This kinematic region is best accessible at lower energies in the center-of-mass system (cms) and/or large rapidities. Therefore, a fixed target experiment like AFTER@LHC [1-4] operating at cms energy $\sqrt{s}=115 \mathrm{GeV}$ with a high luminosity is ideally suited for searches of IC effects. In the following we review some of the collider processes which have been studied in the literature in this respect.

5.1. Open Heavy Flavor Production. Inclusive charm hadron $\left(D^{0}, D^{+}, D^{\star+}, \Lambda_{c}, \ldots\right)$ production in hadronic collisions was advocated in [49] as a laboratory to probe IC inside the colliding hadrons. In this analysis, predictions for the differential cross section in dependence on the transverse momentum $p_{T}$ were obtained in the general-mass variable-flavor-number scheme (GM-VFNS) [50-52] at next-to-leading order (NLO). In this scheme, the charm quark is an active parton and the differential cross sections of inclusive charm meson production depend heavily on the PDF of the charm quark. The sensitivity of these cross sections to IC was studied for the Tevatron at cms energy of $1960 \mathrm{GeV}$ and the Relativistic Heavy Ion Collider (RHIC) at cms energies of $200 \mathrm{GeV}$ (RHIC200) and $500 \mathrm{GeV}$ (RHIC500). The different IC models from the CTEQ6.5c global analysis [40] were employed together with the fragmentation functions for charm mesons from [53]. While the effects at the Tevatron were found to be very moderate and likely not testable, large enhancements were found at RHIC200 reaching values of $\sim 3$ at $p_{T}=20 \mathrm{GeV}$. Unfortunately, the measurements at RHIC200 are limited by the luminosity. At RHIC500 the cross section is increased by about a factor 3.6. However, the sensitivity to IC for the lightfront models is greatly reduced.

More recently, the GM-VFNS was applied to obtain predictions for the production of inclusive $D$ mesons at the LHC for cms energy of $7 \mathrm{TeV}$ (LHC7) [54]. It was found that the production cross sections at large rapidities $y \geq 4$ are sensitive to an IC component. These predictions can be tested by measurements at forward rapidities with the $\mathrm{LHCb}$ detector. 


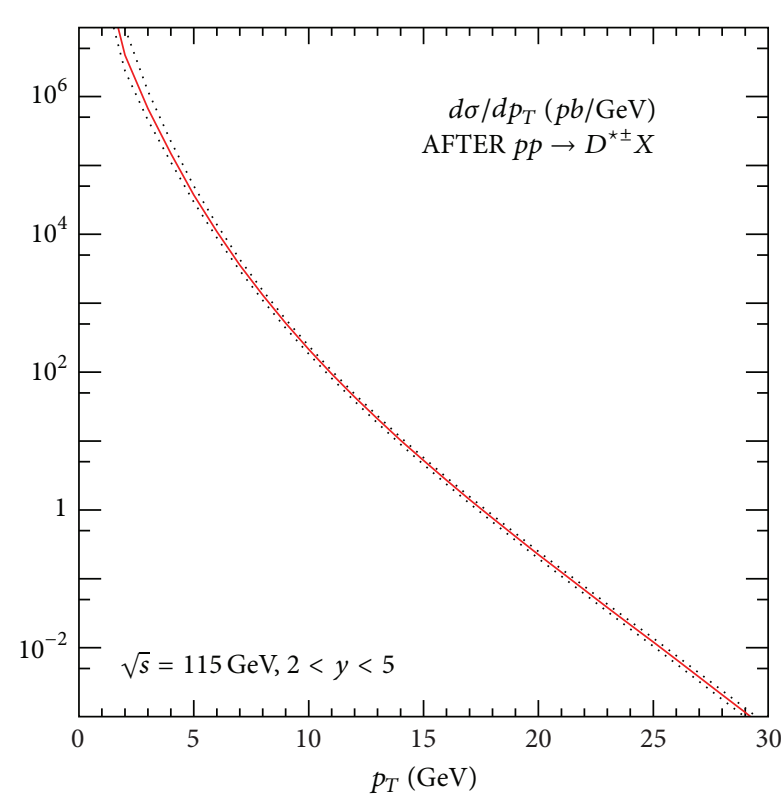

(a)

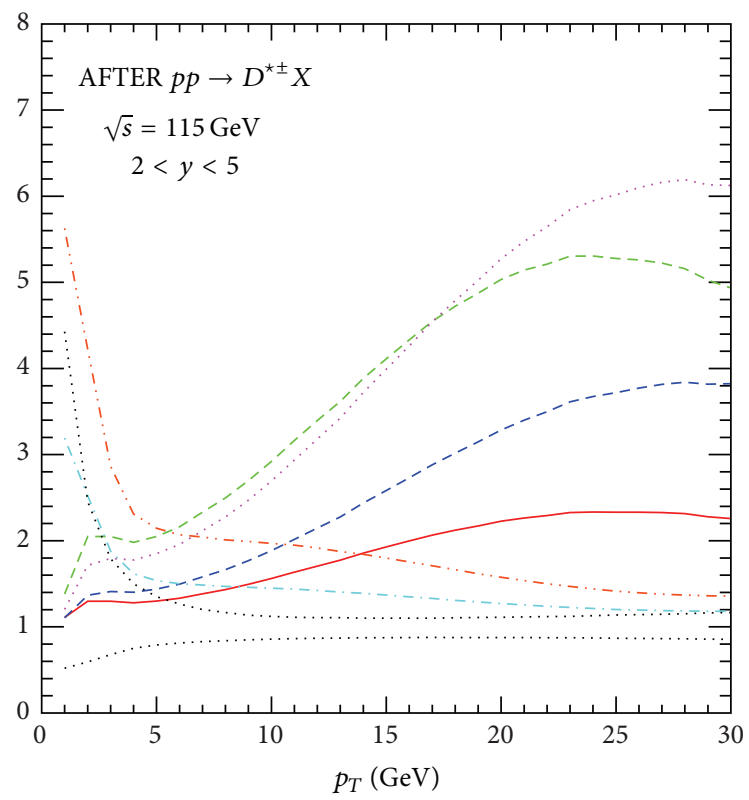

(b)

FiguRE 6: NLO predictions for inclusive $D^{\star}$ meson production at AFTER@LHC versus the transverse momentum of the $D$ meson. (a) Differential cross section on an absolute scale without intrinsic charm. (b) Ratio with respect to to the central prediction of (a). Shown are results using the IC parametrizations from [40] for $n=1$ (red, solid line), 2 (violet, dotted line), 3 (blue, dashed line), 4 (green, long dashed line), 5 (cyan, dot-dashed line), and 6 (orange, double-dot-dashed line). In both figures, the black dotted lines have been obtained by varying the renormalization scale around the central choice $\left(\mu_{R}=m_{T}\right)$ to $\mu_{R}=m_{T} / 2$ (upper line) and $\mu_{R}=2 m_{T}$ (lower line).

The ideal experiment to search for the effects of IC would be a high luminosity fixed target experiment such as AFTER@LHC operating at cms energy of $115 \mathrm{GeV}$. In Figure 6 we show results for inclusive $D^{\star}$ meson production as a function of the transverse momentum of the $D^{\star}$ meson integrated over the rapidity range $2<y<5$ (in the laboratory frame) in essentially the same setup as in [49] to which we refer for details. The only difference is that, following [55], the default choice for the renormalization and factorization scales is $\mu_{R}=m_{T}, \mu_{F}=\mu_{F}^{\prime}=m_{T} / 2$, where $m_{T}=\sqrt{p_{T}^{2}+m^{2}}$ is the transverse mass. The theoretical predictions are shown on an absolute scale in Figure 6(a) and as a ratio with respect to the default results in Figure 6(b). In both figures, the black dotted lines have been obtained by varying the renormalization scale around the central choice to $\mu_{R}=m_{T} / 2$ (upper line) and $\mu_{R}=$ $2 m_{T}$ (lower line). In Figure 6(b) we repeat the calculation of the central prediction in turn with PDF sets CTEQ6.5Cn for $n=1, \ldots, 6$ and normalize the outcome to the default prediction with zero IC of Figure 6(a). We observe that the ratios for $n=1,2,3,4$ corresponding to the BHPS $(n=1,2)$ or meson cloud $(n=3,4)$ models become very large at large $p_{T}$. Indeed, the default cross section can be increased by more than a factor 5 at $p_{T}=20 \mathrm{GeV}$ in scenarios with maximally allowed intrinsic charm $(n=2,4)$. Even for the IC sets with smaller normalization $(n=1,3)$ corresponding to $\langle x\rangle_{c_{1}+\bar{c}_{1}}=0.57 \%$ and $\langle x\rangle_{c_{1}+\bar{c}_{1}}=0.96 \%$ the cross section would be enhanced by a factor larger than 2 (red solid line) or 3 (blue dashed line) at $p_{T}=20 \mathrm{GeV}$. It is also interesting to note that the phenomenological models for a sea-like IC $(n=$ $5,6)$ lead to a significant enhancement of the cross section at small $p_{T} \sim m_{c}$ which would be probed at AFTER@LHC as well.

5.2. Production of a Photon in Association with a Charm Quark. Another process with a wide range of phenomenological applications in $p p, p A$, and $A A$ collisions [56-58] which is very sensitive to the heavy quark PDF is the associated production of a photon with a heavy quark. A dedicated study of this process at the LHC operating at $\sqrt{s}=8 \mathrm{TeV}$ (LHC8) was performed in $[59,60]$ where it was demonstrated that the existence of IC in the proton can be visible at large transverse momenta of the photons and heavy quark jets at rapidities $1.5<\left|y_{\gamma}\right|<2.4,\left|y_{c}\right|<2.4$. Indeed, for the BHPS model the cross section can be enhanced by a factor of 2-3 for $p_{T}^{\gamma}>200 \mathrm{GeV}$ (see Figure 5 in [60]). This comes with the penalty that the cross section falls rapidly with increasing transverse momentum so that this measurement will be limited by statistics.

Again, as for open heavy flavor production, the lower $\mathrm{cms}$ energy together with the high luminosity makes a fixed target experiment like AFTER@LHC the ideal place to discover IC using $\gamma+c$ production. This can be seen in Figure 7, where the differential cross section is enhanced by a factor 5 at $p_{T}^{\gamma}=$ $20 \mathrm{GeV}$ (b) with a not too small cross section (a).

5.3. Vector Boson Production. Dulat et al. [42] studied the sensitivity of $W^{ \pm}$and $Z^{0}$ production to the presence of IC. Vector boson production at the LHC is an interesting testing ground for IC because they are produced at relatively large $x$ and $Z^{0} \rightarrow l^{+} l^{-}$is a rather clean final state. They did a NNLO 


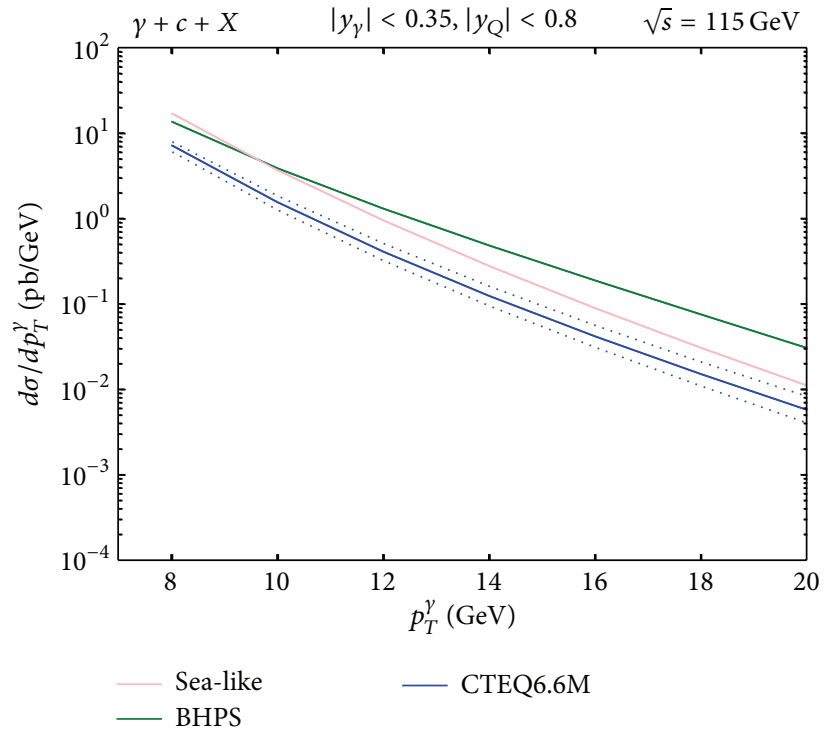

(a)

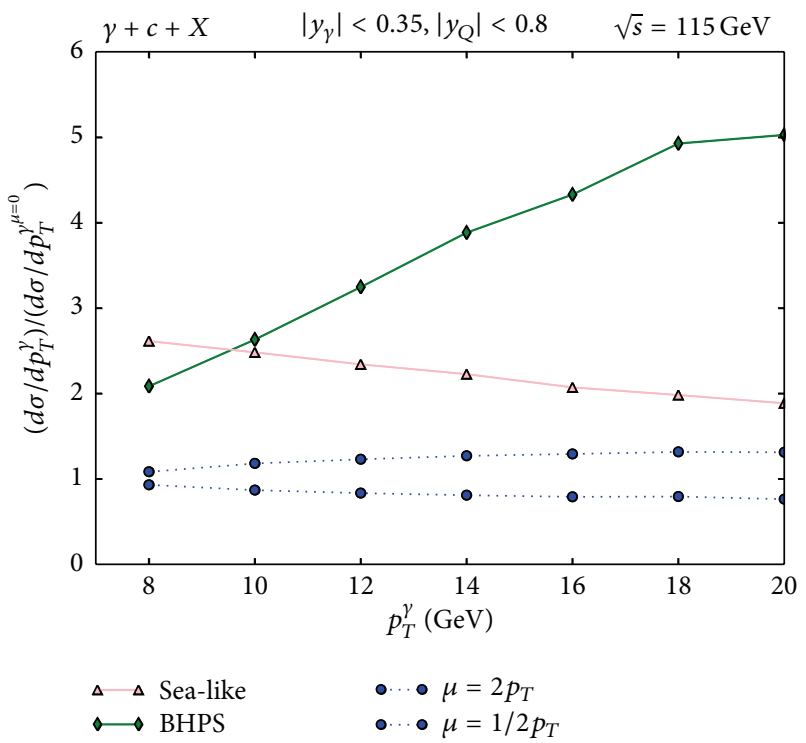

(b)

FIGURE 7: (a) NLO predictions for the production of a prompt photon in association with a charm quark jet in $p p$ collisions at AFTER@LHC versus the transverse momentum of the photon. Shown are results for a BHPS and a sea-like intrinsic charm using the CTEQ6.6c PDFs. For comparison, the predictions without an IC using the CTEQ6.6M PDFs are shown as well together with the uncertainty band obtained by varying the central factorization scale $\mu_{F}=p_{T}^{\gamma}$ by a factor 2 up and down (blue, dotted curves). (b) Depicting the ratio of the curves in (a) with respect to the central prediction without intrinsic charm.

calculation of $W$ and $Z$ production including IC based on their global fits at $\sqrt{s}=8$ and $14 \mathrm{TeV}$. They also studied the ratio $d \sigma_{W^{+}+W^{-}}(y) / d \sigma_{Z^{0}}(y)$ relative to the result with no IC. Neither of these calculations showed an effect larger than the uncertainties due to the CT10 sets themselves. However, when the $Z^{0} p_{T}$ distribution with IC was compared to that without, they saw a factor of two enhancement at $p_{T} \sim 500 \mathrm{GeV}$ for $\sqrt{s}=8 \mathrm{TeV}$ in the range $|\eta|<2.1$. The corresponding enhancement at $14 \mathrm{TeV}$ was smaller at the same $p_{T}$ because the $x$ value reached is reduced at the higher energy [42].

We show a simple test case here for $W$ and $Z$ production to $\mathrm{NLO}$ at $\sqrt{s}=7 \mathrm{TeV}$. We use only the BHPS IC parameterization for the five-particle Fock state, shown in (10). We assume a $1 \%$ normalization and no $Q^{2}$ evolution to maximize the possible effect at forward rapidity. The $p_{T^{-}}$ integrated rapidity distribution is shown in Figure 8, as is the ratio of the result with IC to that without as a function of rapidity. The rapidity distributions without IC are given by the solid curves while the dashed curves are the calculations with the BHPS IC contribution to the charm parton density. With BHPS IC, one expects enhancement only at forward rapidity. The enhancement from IC appears for $|y|>2.5$. Note that if the sea-like IC would be used instead, the enhancement would be small but finite over all rapidity.

The $W^{+}$cross section is largest and most forward peaked, because of the $u \bar{d}$ contribution. The contribution from the $c \bar{d}$ part is a very small addition since the $u$ valence contribution is large and peaks at large $x$, making the $y$ distribution larger at $|y| \sim 2$ than at $y=0$. Indeed, it gives the smallest IC contribution. The $W^{-}$distribution should have the largest possible contribution from IC because both $d \bar{u}$ and $d \bar{c}$ peak at low $x$ and because the $d$ valence distribution peaks at lower $x$ so that the $W^{-}$rapidity distribution has a maximum at $y=0$. At $|y| \sim 4$, the IC enhancement is $\sim 40 \%$. Finally, the $Z^{0}$ distribution, with a plateau over $|y|<1.5$, also has a very small IC contribution because the charm enhancement only comes through $c \bar{c}$.

Such IC enhancements are only visible outside the midrapidity acceptance of the collider detector coverage of CMS and ATLAS. However, LHCb or ALICE covers this forward rapidity range with muons and could detect forward $Z^{0}$. They could also look at the lepton rapidity asymmetry $\left(W^{+}-\right.$ $\left.W^{-}\right) /\left(W^{+}+W^{-}\right)$at forward rapidity. The statistical accuracy of the measurement would need to be high to distinguish an IC enhancement from the no IC result, especially since the $1 \%$ BHPS IC is likely an upper limit on this enhancement. Note that the higher energy of LHC Run 2 will reduce the potential enhancement even though it would increase the rates.

\section{Conclusions}

The existence of nonperturbative intrinsic charm and bottom components is a fundamental prediction of QCD. In this paper, we have reviewed the current status of our understanding of this intrinsic heavy quark content of the nucleon which yet remains to be confirmed experimentally. In particular, after introducing theoretical models predicting the intrinsic heavy quark distributions we have turned to a summary of the available information on intrinsic charm coming from global analyses of parton distribution functions. There are no global analyses of intrinsic bottom available and we have described how IB can be modeled in order to explore its impact on collider observables keeping in mind that bottom quark initiated subprocesses play an important 


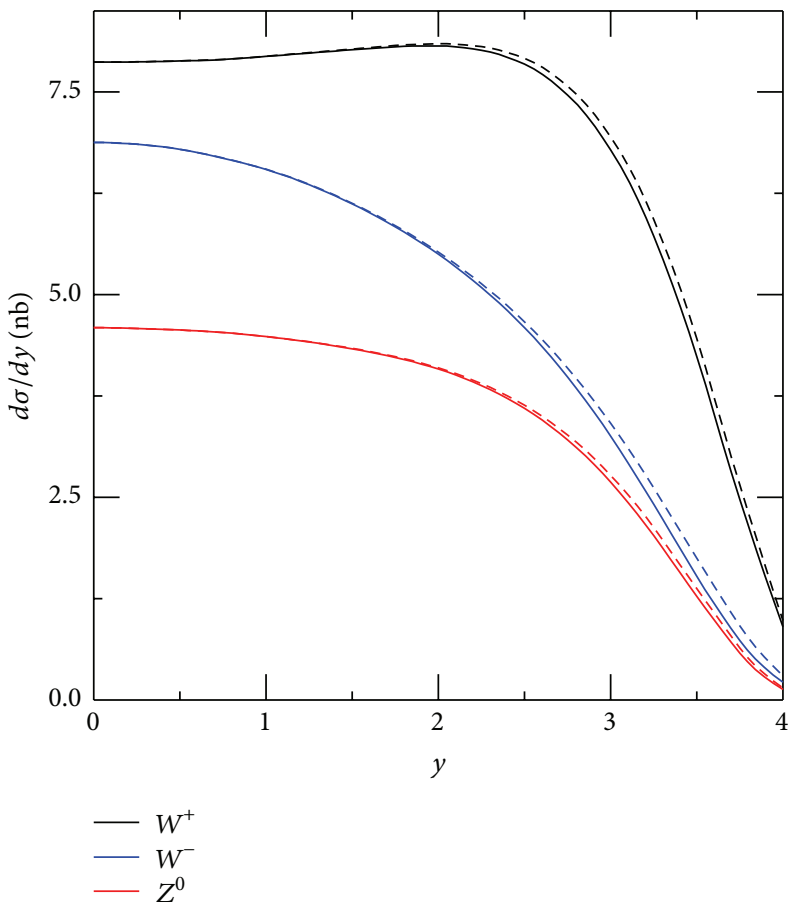

(a)

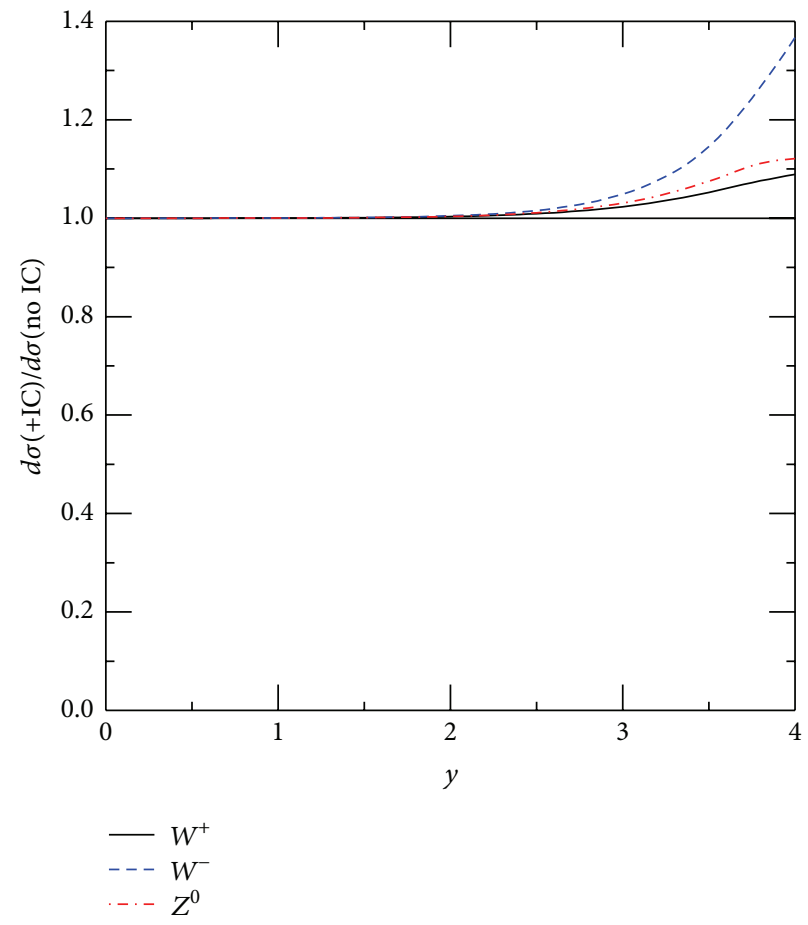

(b)

FIgure 8: The $W^{+}$(black), $W^{-}$(blue), and $Z^{0}$ (red) rapidity distributions (a). The solid curves are the results without IC while the dashed curves include $1 \%$ BHPS IC. The ratios of the dashed curves to the solid curves showing the enhancement of the rapidity distributions due to IC for $W^{+}$(solid black), $W^{-}$(blue dashed), and $Z^{0}$ (red dot dashed) are shown in (b).

role in certain electroweak observables and in models for physics beyond the Standard Model. We then have turned to a discussion of collider processes where IC could be discovered. Generally, the effects of IC are larger at colliders with a lower center-of-mass energy and for hard processes with moderate factorization scales. Therefore, a high luminosity fixed target experiment like AFTER@LHC operating at a center-of-mass energy $\sqrt{s}=115 \mathrm{GeV}$ would be ideally suited to discover or constrain IC.

\section{Conflict of Interests}

The authors declare that there is no conflict of interests regarding the publication of this paper.

\section{Acknowledgments}

The authors are grateful to T. Stavreva for providing Figure 7. The work of S. J. Brodsky was supported by the Department of Energy Contract no. DE-AC02-76SF00515. The work of R. Vogt was performed under the auspices of the US Department of Energy by Lawrence Livermore National Laboratory under Contract DE-AC52-07NA27344.

\section{References}

[1] S. J. Brodsky, F. Fleuret, C. Hadjidakis, and J. P. Lansberg, "Physics opportunities of a fixed-target experiment using LHC beams," Physics Reports, vol. 522, no. 4, pp. 239-255, 2013.
[2] J. P. Lansberg, S. J. Brodsky, F. Fleuret, and C. Hadjidakis, "Quarkonium physics at a fixed-target experiment using the LHC Beams," Few-Body Systems, vol. 53, no. 1-2, pp. 11-25, 2012.

[3] J. Lansberg, R. Arnaldi, S. Brodsky et al., "AFTER@LHC: a precision machine to study the interface between particle and nuclear physics," EPJ Web of Conferences, vol. 66, Article ID 11023, 4 pages, 2014.

[4] A. Rakotozafindrabe, M. Anselmino, R. Arnaldi et al., "Studying the high $x$ frontier with afixed-target experiment at the LHC," in Proceedings of the 21st International Workshop on Deep-Inelastic Scattering and Related Subject (DIS '13), p. 250, Marseille, France, April 2013.

[5] S. J. Brodsky, P. Hoyer, C. Peterson, and N. Sakai, "The intrinsic charm of the proton," Physics Letters B, vol. 93, no. 4, pp. 451455, 1980.

[6] S. J. Brodsky, J. C. Collins, S. D. Ellis, J. F. Gunion, and A. H. Mueller, "Intrinsic chevrolets at the SSC", in Proceedings of the 1984 Summer Study on the SSC, Snowmass, Colo, USA, JuneJuly 1984, Edited by R. Donaldson and J. G. Morfin (AIP, New York, NY, USA, 1985).

[7] B. Harris, J. Smith, and R. Vogt, "Reanalysis of the EMC charm production data with extrinsic and intrinsic charm at NLO," Nuclear Physics B, vol. 461, pp. 181-196, 1996.

[8] M. Franz, M. V. Polyakov, and K. Goeke, "Heavy quark mass expansion and intrinsic charm in light hadrons," Physical Review D, vol. 62, Article ID 074024, 2000.

[9] W. Freeman and D. Toussaint, "Intrinsic strangeness and charm of the nucleon using improved staggered fermions," Physical Review D, vol. 88, no. 5, Article ID 054503, 18 pages, 2013. 
[10] A. Kryjevski, "Heavy quark $\bar{q} q$ matrix elements in the nucleon from perturbative QCD," Physical Review D, vol. 70, Article ID 094028, 2004.

[11] J. J. Aubert, G. Bassompierre, K. H. Becks et al., "Production of charmed particles in $250 \mathrm{GeV} \mu^{+}$-iron interactions," Nuclear Physics B, vol. 213, no. 1, pp. 31-64, 1983.

[12] G. Bari, M. Basile, G. Bruni et al., "The $\Lambda_{b}^{0}$ beauty baryon production in proton-proton interactions at $\sqrt{s}=62 \mathrm{GeV}$ : a second observation," Il Nuovo Cimento A, vol. 104, no. 12, pp. 1787-1800, 1991.

[13] R. Vogt, S. J. Brodsky, and P. Hoyer, "Systematics of charm production in hadronic collisions," Nuclear Physics B, vol. 383, no. 3, pp. 643-684, 1992.

[14] R. Vogt and S. J. Brodsky, "Charmed hadron asymmetries in the intrinsic charm coalescence model," Nuclear Physics B, vol. 478, no. 1-2, pp. 311-332, 1996.

[15] T. Gutierrez and R. Vogt, "Leading charm in hadron-nucleus interactions in the intrinsic charm model," Nuclear Physics B, vol. 539, pp. 189-214, 1999.

[16] R. Vogt, S. J. Brodsky, and P. Hoyer, "Systematics of $J / \psi$ production in nuclear collisions," Nuclear Physics B, vol. 360, no. 1, pp. 67-96, 1991.

[17] P. Hoyer, M. Vanttinen, and U. Sukhatme, "Violation of factorization in charm hadroproduction," Physics Letters B, vol. 246, pp. 217-220, 1990.

[18] R. Vogt and S. J. Brodsky, "Intrinsic charm contribution to double quarkonium hadroproduction," Physics Letters B, vol. 349, no. 4, pp. 569-575, 1995.

[19] S. J. Brodsky, B. Kopeliovich, I. Schmidt, and J. Soffer, "Diffractive Higgs production from intrinsic heavy flavors in the proton," Physical Review D, vol. 73, no. 11, Article ID 113005, 13 pages, 2006.

[20] S. J. Brodsky, A. S. Goldhaber, B. Z. Kopeliovich, and I. Schmidt, "Higgs hadroproduction at large Feynman $x$," Nuclear Physics B, vol. 807, no. 1-2, pp. 334-347, 2009.

[21] S. J. Brodsky and S. Gardner, "Evading the CKM hierarchy: intrinsic charm in $B$ decays," Physical Review D, vol. 65, Article ID 054016, 2002.

[22] S. Brodsky, G. de Teramond, and M. Karliner, "Puzzles in hadronic physics and novel quantum chromodynamics phenomenology," Annual Review of Nuclear and Particle Science, vol. 62, pp. 1-35, 2012.

[23] S. J. Brodsky, C. Peterson, and N. Sakai, "Intrinsic heavy-quark states," Physical Review D, vol. 23, no. 11, p. 2745, 1981.

[24] S. J. Brodsky, P. Hoyer, A. H. Mueller, and W.-K. Tang, "New QCD production mechanisms for hard processes at large $x$," Nuclear Physics B, vol. 369, no. 3, pp. 519-542, 1992.

[25] S. Paiva, M. Nielsen, F. S. Navarra, F. O. Duraes, and L. L. Barz, "Virtual meson cloud of the nucleon and intrinsic strangeness and charm," Modern Physics Letters A, vol. 13, no. 34, pp. 27152724, 1998.

[26] M. Neubert, "Heavy-quark symmetry," Physics Reports, vol. 245, no. 5-6, pp. 259-395, 1994.

[27] F. M. Steffens, W. Melnitchouk, and A. W. Thomas, "Charm in the nucleon," The European Physical Journal C-Particles and Fields, vol. 11, no. 4, pp. 673-683, 1999.

[28] J. Pumplin, "Light-cone models for intrinsic charm and bottom," Physical Review D, vol. 73, Article ID 114015, 2006.

[29] T. Hobbs, J. Londergan, and W. Melnitchouk, "Phenomenology of nonperturbative charm in the nucleon," Physical Review D, vol. 89, Article ID 074008, 2014.
[30] P. Chauvat, R. Cousins, K. Hayes et al., "Production of $\Lambda_{c}$ with large $X_{\mathrm{F}}$ at the isr," Physics Letters B, vol. 199, no. 2, pp. 304-310, 1987.

[31] F. Garcia, G. Alkhazov, A. G. Atamantchouk et al., "Hadronic production of $\Lambda_{c}$ from $600 \mathrm{GeV} / c \pi^{-}, \Sigma^{-}$and p beams," Physics Letters B, vol. 528, no. 1-2, pp. 49-57, 2002.

[32] G. Altarelli and G. Parisi, "Asymptotic freedom in parton language," Nuclear Physics B, vol. 126, pp. 298-318, 1977.

[33] V. N. Gribov and L. N. Lipatov, "Deep inelastic ep scattering in perturbation theory," Soviet Journal of Nuclear Physics, vol. 15, p. $438,1972$.

[34] Y. L. Dokshitzer, "Calculation of the structure functions for deep inelastic scattering and $\mathrm{e}^{+} \mathrm{e}^{-}$annihilation by perturbation theory in quantum chromodynamics," Soviet Physics-JETP, vol. 46, pp. 641-653, 1977.

[35] J. C. Collins and W.-K. Tung, "Calculating heavy quark distributions," Nuclear Physics B, vol. 278, no. 4, pp. 934-950, 1986.

[36] M. Buza, Y. Matiounine, J. Smith, and W. L. van Neerven, "Charm electroproduction viewed in the variable-flavour number scheme versus fixed-order perturbation theory," The European Physical Journal C, vol. 1, no. 1-2, pp. 301-320, 1998.

[37] A. Belyaev, J. Pumplin, W.-K. Tung, and C. P. Yuan, "Uncertainties of the inclusive Higgs production cross section at the tevatron and the LHC," Journal of High Energy Physics, vol. 2006, no. 1, article 069, 2006.

[38] F. Lyonnet, A. Kusina, T. Ježo et al., "On the intrinsic bottom content of the nucleon and its impact on heavy new physics at the LHC," Journal of High Energy Physics, http://arxiv.org/abs/1504.05156.

[39] E. Hoffmann and R. Moore, "Sub-leading contributions to the intrinsic charm of the nucleon," Zeitschrift für Physik C, vol. 20, no. 1, pp. 71-82, 1983.

[40] J. Pumplin, H. L. Lai, and W. K. Tung, "Charm parton content of the nucleon," Physical Review D, vol. 75, Article ID 054029, 2007.

[41] P. M. Nadolsky, H.-L. Lai, Q.-H. Cao et al., "Implications of CTEQ global analysis for collider observables," Physical Review $D$, vol. 78, no. 1, Article ID 013004, 21 pages, 2008.

[42] S. Dulat, T.-J. Hou, J. Gao et al., "Intrinsic charm parton distribution functions from CTEQ-TEA global analysis," Physical Review D, vol. 89, no. 7, Article ID 073004, 18 pages, 2014.

[43] P. Jimenez-Delgado, T. J. Hobbs, J. T. Londergan, and W. Melnitchouk, "New limits on intrinsic charm in the nucleon from global analysis of parton distributions," Physical Review Letters, vol. 114, Article ID 082002, 2015.

[44] P. Jimenez-Delgado and E. Reya, "Delineating parton distributions and the strong coupling," Physical Review D, vol. 89, no. 7, Article ID 074049, 17 pages, 2014.

[45] S. J. Brodsky and S. Gardner, "Comment on 'New limits on intrinsic charm in the nucleon from global analysis of parton distributions', http://arxiv.org/abs/1504.00969.

[46] P. Jimenez-Delgado, T. J. Hobbs, J. T. Londergan, and W. Melnitchouk, "Reply to comment on 'new limits on intrinsic charm in the nucleon from global analysis of parton distributions', http://arxiv.org/abs/1504.06304.

[47] D. Stump, J. Huston, J. Pumplin et al., "Inclusive jet production, parton distributions, and the search for new physics," Journal of High Energy Physics, vol. 2003, no. 10, article 046, 2003.

[48] P. M. Nadolsky and Z. Sullivan, "PDF uncertainties in WH production at Tevatron," http://arxiv.org/abs/hep-ph/0110378. 
[49] B. A. Kniehl, G. Kramer, I. Schienbein, and H. Spiesberger, "Open charm hadroproduction and the charm content of the proton," Physical Review D, vol. 79, no. 9, Article ID 094009, 10 pages, 2009.

[50] B. A. Kniehl, G. Kramer, I. Schienbein, and H. Spiesberger, "Inclusive $D^{* \pm}$ production in $p \bar{p}$ collisions with massive charm quarks," Physical Review D, vol. 71, Article ID 014018, 2005.

[51] B. A. Kniehl, G. Kramer, I. Schienbein, and H. Spiesberger, "Collinear subtractions in hadroproduction of heavy quarks," The European Physical Journal C-Particles and Fields, vol. 41, no. 2, pp. 199-212, 2005.

[52] B. A. Kniehl, G. Kramer, I. Schienbein, and H. Spiesberger, "Hadroproduction of D and B mesons in a massive VFNS," in Proceedings of the 13th International Workshop on Deep Inelastic Scattering (DIS '05), Madison, Wis, USA, April 2005.

[53] T. Kneesch, B. Kniehl, G. Kramer, and I. Schienbein, "Charmedmeson fragmentation functions with finite-mass corrections," Nuclear Physics B, vol. 799, no. 1-2, pp. 34-59, 2008.

[54] B. Kniehl, G. Kramer, I. Schienbein, and H. Spiesberger, "Inclusive charmed-meson production at the CERN LHC," The European Physical Journal C, vol. 72, article 2082, 2012.

[55] B. Kniehl, G. Kramer, I. Schienbein, and H. Spiesberger, "Inclusive $B$-meson production at small $p_{T}$ in the general-mass variable-flavor-number scheme," The European Physical Journal C, vol. 75, p. 140, 2015.

[56] T. P. Stavreva and J. F. Owens, "Direct photon production in association with a heavy quark at hadron colliders," Physical Review D, vol. 79, Article ID 054017, 2009.

[57] T. Stavreva, I. Schienbein, F. Arleo et al., "Probing gluon and heavy-quark nuclear PDFs with $\gamma+\mathrm{Q}$ production in $\mathrm{pA}$ collisions," Journal of High Energy Physics, vol. 2011, no. 1, article 152, 2011.

[58] T. Stavreva, F. Arleo, and I. Schienbein, "Prompt photon in association with a heavy-quark jet in $\mathrm{Pb}-\mathrm{Pb}$ collisions at the LHC," Journal of High Energy Physics, vol. 2013, no. 2, article 072, 2013.

[59] V. Bednyakov, M. Demichev, G. Lykasov, T. Stavreva, and M. Stockton, "Searching for intrinsic charm in the proton at the LHC," EPJ Web of Conferences, vol. 60, Article ID 20047, 3 pages, 2013.

[60] V. A. Bednyakov, M. A. Demichev, G. I. Lykasov, T. Stavreva, and M. Stockton, "Searching for intrinsic charm in the proton at the LHC," Physics Letters, Section B: Nuclear, Elementary Particle and High-Energy Physics, vol. 728, no. 1, pp. 602-606, 2014. 

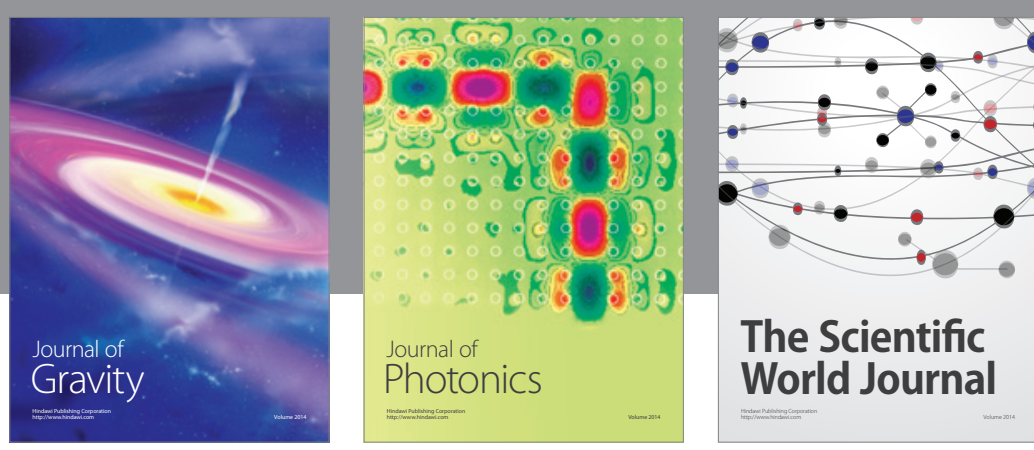

The Scientific World Journal
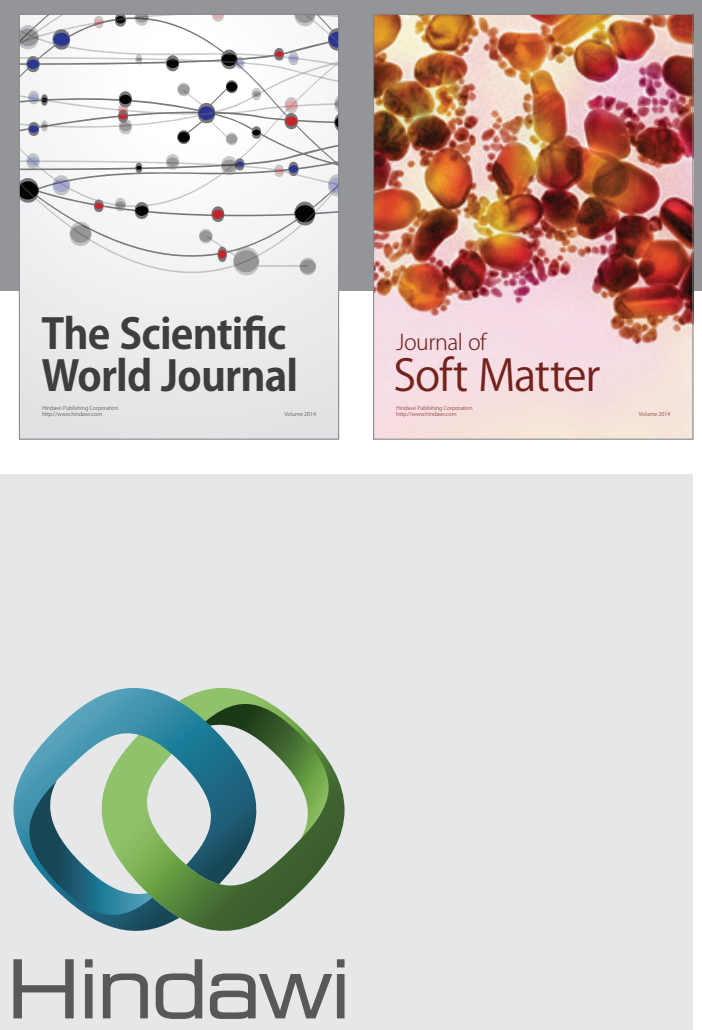

Submit your manuscripts at

http://www.hindawi.com

nternational Journal of

Statistical Mechanics
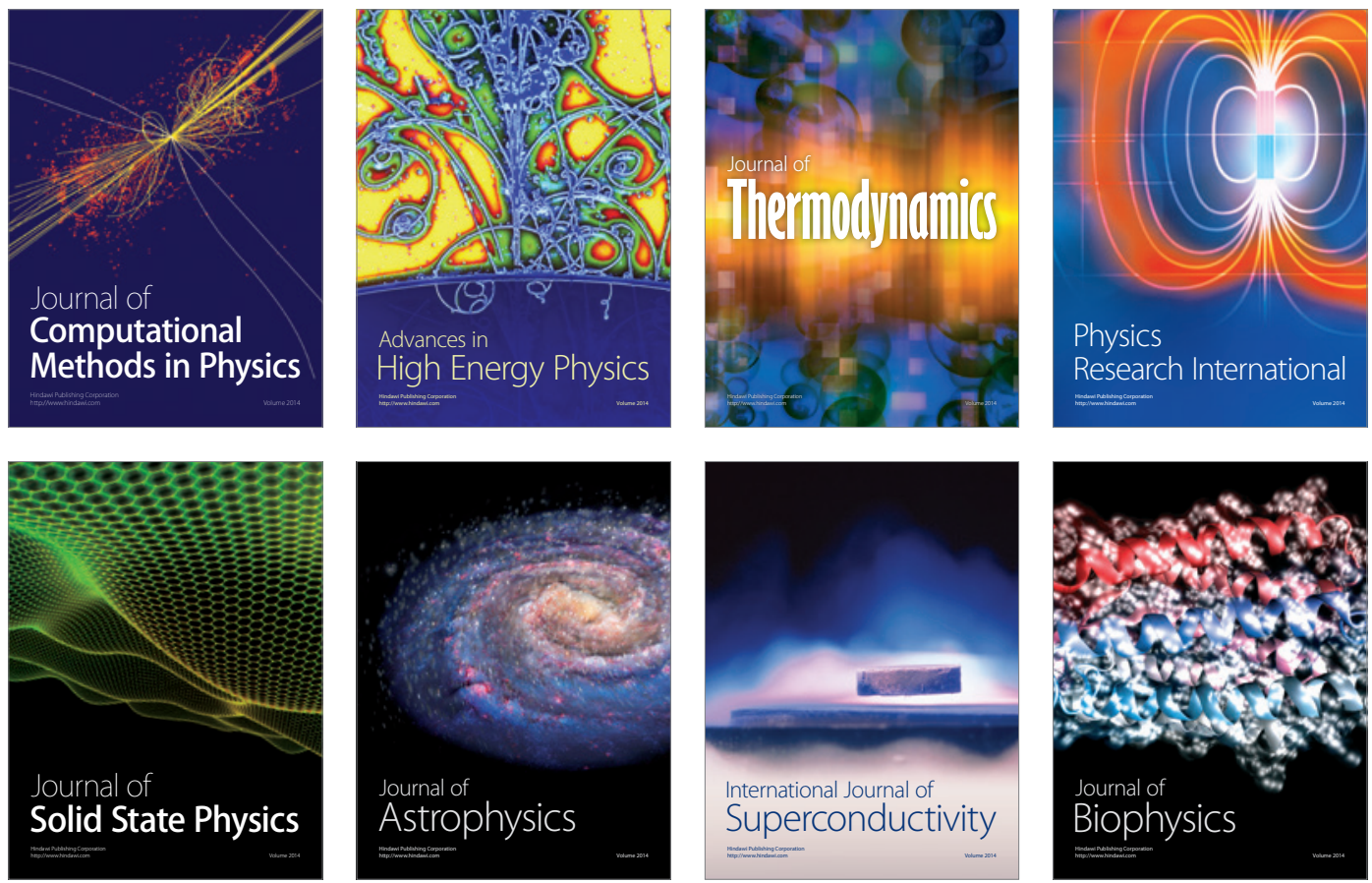
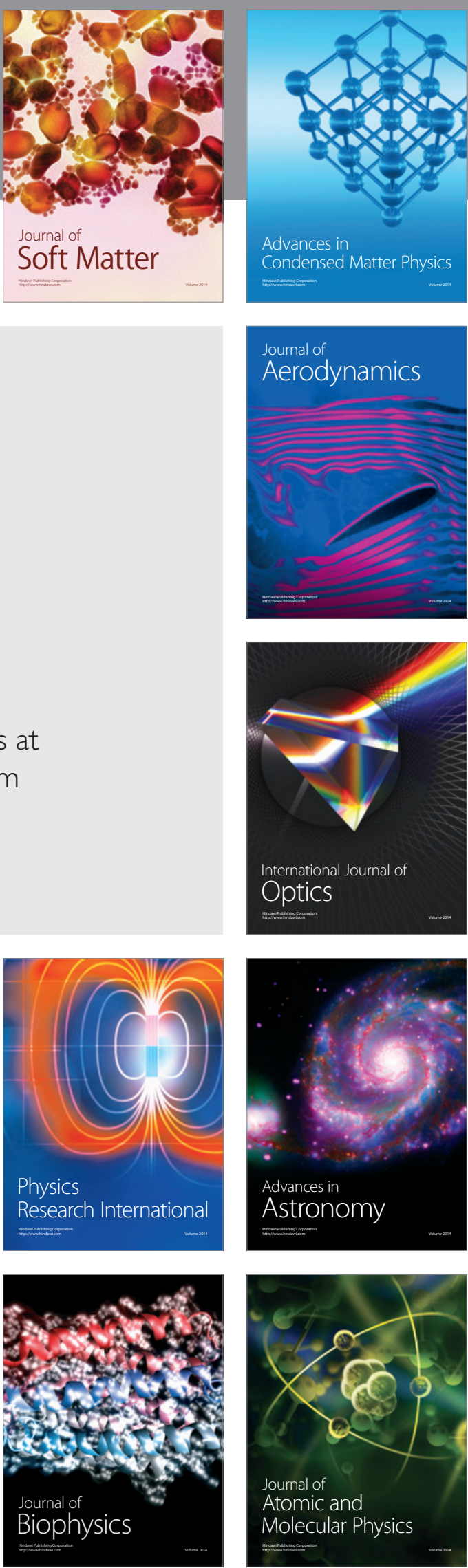